\title{
GESTÃO HOSPITALAR: OS DESAFIOS NA IMPLANTAÇÃO COM QUALIDADE
}

\section{GESTIÓN HOSPITALARIA: LOS RETOS DE LA IMPLANTACIÓN DE CALIDAD}

\section{HOSPITAL MANAGEMENT: THE CHALLENGES IN QUALITY IMPLEMENTATION}

\author{
Jessianie Aparecida Ávila BANDEIRA \\ Centro Universitário Internacional (UNINTER) - Polo Altamira - Pará \\ Graduanda em Bacharel em Administração (2016 - 2020) \\ ORCID: https://orcid.org/0000-0002-8174-9755 \\ E-mail: ci_atm@hotmail.com \\ Marconde Ávila BANDEIRA \\ Universidade Federal do Pará (UFPA) Belém - PA - Brasil. \\ Mestrando Núcleo de Estudos Transdisciplinares em Educação Básica. \\ Programa de Pós-graduação em Currículo e Gestão da Escola Básica. Pedagogo. \\ ORCID: https://orcid.org/0000-0001-8414-0761 \\ E-mail: bandeira.neto77@gmail.com
}




\section{RESUMO}

O presente artigo tem como objetivo mostrar os desafios da gestão com qualidade na prestação de serviços da área hospitalar que faz parte do nível hierárquico terciário da Rede de Atenção em Saúde Especializada de Média e Alta Complexidade. Realizamos revisão bibliográfica nos sites da Organização Nacional de Acreditação (ONA), da Associação Beneficente de Assistência Social Hospitalar (Pró-Saúde), do Google e em livros específicos da área. A estrutura do artigo traz quatro tópicos: 1) Introdução; 2) Resultados e discussões; 3) Considerações finais e nossas referências. É possível constatarmos no Brasil o sucesso da busca por entendimento entre as partes envolvidas na área hospitalar, pois tem sido crescente o reconhecimento de instituições que tem êxito nos processos de acreditação.

Palavras-Chave: Qualidade. Gestão hospitalar. Organização nacional de acreditação.

\section{RESEUMEN}

Este artículo tiene como objetivo mostrar los desafíos de la gestión de la calidad en la prestación de servicios en el área hospitalaria que forma parte del nivel jerárquico terciario de la Red de Atención Especializada de Salud de Complejidad Media y Alta. Realizamos una revisión bibliográfica en los sitios web de la Organização Nacional de Acreditação (ONA), la Asociación Benéfica de Asistencia Social Hospitalaria (Pró-Saúde), Google y en libros específicos del área. La estructura del artículo tiene cuatro temas: 1) Introducción; 2) Resultados y discusiones; 3) Consideraciones finales y nuestras referencias. Es posible verificar en Brasil el éxito de la búsqueda de entendimiento entre las partes involucradas en el área hospitalaria, ya que ha habido un creciente reconocimiento de instituciones que tienen éxito en los procesos de acreditación.

Palabras Clave: Calidad. Gestión hospitalaria. Organização nacional de acreditação.

\section{ABSTRACT}

This article aims to show the challenges of quality management in the provision of services in the hospital area that is part of the tertiary hierarchical level of the Medium and High Complexity Specialized Health Care Network. We performed a bibliographic review on the websites of the Organização Nacional de Acreditação (ONA), the Beneficent Association of Hospital Social Assistance (Pró-Saúde), Google and on specific books in the area. The structure of the article has four topics: 1) Introduction; 2) Results and discussions; 3) Final considerations and our references. It is possible to verify in Brazil the success of the search for understanding between the parties involved in the hospital area, as there has been a growing recognition of institutions that are successful in the accreditation processes.

Keywords: Quality. Hospital management. Organização nacional de acreditação. 
BANDEIRA, J.A.A; BANDEIRA, M. A., Gestão Hospitalar: os desafios na implantação com qualidade. R. Científica UBM - Barra Mansa (RJ), ano XXVI, v. 23, n. 44, 1 . Sem. 2021 p. 103-114.

ISSN 1516-4071

\section{INTRODUÇÃO}

Na década de 1980 - primordialmente após a VIII Conferência Nacional de Saúde e concomitante com a realização da Assembleia Nacional Constituinte, ambas realizadas em 1986, no Distrito Federal, cidade de Brasília - houve avanços na redemocratização do Estado brasileiro, com a inserção das demandas vindouras das discussões realizadas pela sociedade civil organizada, destacando os direitos sociais como políticas públicas essenciais, tais como a seguridade social, a educação, a saúde, o emprego, o saneamento, a habitação e a estrutura fundiária, posteriormente promulgados e garantidos na Constituição Federal de 1988 (BRASIL, 1988 ).

Nesse contexto, a saúde vivencia fortes transformações em direção a descentralização com a promulgação das leis orgânicas da saúde, as quais são: Lei número 8.080, de 19 de setembro de 1990, que "Dispões sobre as condições para a promoção, proteção e recuperação da saúde, a organização e o funcionamento dos serviços correspondentes" e a lei número 8.142, de 28 de dezembro de 1990, que "Dispõe sobre a participação da comunidade na gestão do Sistema Único de Saúde e sobre as transferências intergovernamentais de recursos financeiros na área da saúde e dá outras providencias" (BRASIL, 1990).

Nesse sentido, para Brasil e Capela (2016, p. 79), "Entender a valorização e a consolidação das políticas públicas no Brasil" se vincula "aos processos de descentralização e de gerenciamento, típicos da Reforma administrativa ocorrida no Brasil em meados dos anos 1990”. Com isso, os referidos autores, inferem que “A descentralização do poder, a aposta na maior autonomia e responsabilização dos entes Federativos - com destaques para o Municipal”, estão "unidas à questão da maior redemocratização já indicadas na Constituição" e que ainda “surgem como um ponto importante nos estudos das política públicas no Brasil, pósredemocratização" (BRASIL; CAPELA, 2016, p. 82).

Para isso, como citadas anteriormente, as Leis $\mathrm{n}^{\circ} 8.080$ e 8.142 de 1990 vem organizando e regulando o Sistema Único de Saúde com conjuntos de atos e serviços de saúde, proporcionados por organismos e instituições públicas, nas esferas federal, estadual e municipal, da administração direta e indireta e das fundações mantidas pelo poder público, sendo que a iniciativa privada participa em caráter complementar (BRASIL, 1990).

Entretanto, 21 anos depois (2011), o Governo Federal, publica o Decreto número 7.508/2011 que visa a regulação da Lei número 8.080/1990, se dispondo sobre a organicidade 
do SUS, de seu planejamento da saúde, de sua assistência à saúde e a sua articulação interfederativa, dentre outras providências. Tal instrumento regulador exigiu nova dinamicidade organizativa e de gerenciamento para atenção à saúde, marcando a descentralização e regionalização do SUS (BRASIL, 2011).

Contudo, tais atos e serviços na saúde são executados com atenção aos direcionamentos previstos no artigo 198 da Constituição Federal de 1988, correspondendo aos princípios organizativos e doutrinários do Sistema Único de Saúde, os quais são:

- Universalidade de acesso aos serviços de saúde em todos os níveis de assistências;

- Equidade;

- Descentralização político-administrativa com direção única em cada esfera de governo;

- Conjunto de recursos financeiros, tecnológicos, materiais e humanos da união dos Estados, do Distrito Federal e dos Municípios na prestação de serviços de assistência à saúde da população;

- Participação da comunidade;

- Regionalização e hierarquização.

Portanto, mediante tais princípios organizacionais e doutrinadores do Sistema, surge a formulação e redesenho de um extenso programa de descentralizações, principalmente na saúde, e nesse intuito, surgem as normativas formalizando as Redes de Atenção em Saúde que trazemos neste estudo, em específico a Portaria $n^{\circ} 4.279$ de 31 de dezembro de 2010, que vem definir as "Diretrizes para a Organização das Redes de Atenção em Saúde no âmbito do SUS" (BRASIL, 2010), definindo uma rede regionalizada e hierarquizada para o Sistema Único de Saúde (SUS), considerando o nivelamento complexo de funcionamento crescente de atenção primária, secundária e terciária.

Para Sousa e Sousa (2018, p .1), “As Redes de Atenção em Saúde (RAS) são consideradas como arranjos organizacionais que são realizados nas ações e serviços de saúde", com isso é "um sistema de apoio logístico, técnico e de gestão" que visa "garantir a integralidade do cuidado prestado ao usuário dentro do SUS". Com tais arranjos para a organicidade das RAS, ainda de acordo com os autores, surgiram cinco Redes, as quais são: 1) A Rede de Atenção à Urgência e Emergência (RUE); 2) A Rede de Atenção Psicossocial (RAPS); 3) A Rede de Atenção à Pessoas com Doenças Crônicas (RASPDC); 4) A Rede Cegonha (RC) e a 5) A Rede de Cuidados à Saúde da Pessoa com Deficiência.

Nesse caso, como nosso objeto de estudo é a gestão hospitalar com qualidade, buscamos uma maior especificidade da RUE, que para Sousa e Sousa (2018) apud Cyrino (2017) surgiu 
BANDEIRA, J.A.A; BANDEIRA, M. A., Gestão Hospitalar: os desafios na implantação com qualidade. R. Científica UBM - Barra Mansa (RJ), ano XXVI, v. 23, n. 44, 1 . Sem. 2021 p. 103-114.

ISSN 1516-4071

por intermédio da Portaria $\mathrm{n}^{\circ} 1.600$, de 7 de junho de 2011, preconizando assim que o trabalho dos profissionais "seja realizado em conjunto e independente conforme o nível de responsabilidade de cada componente". Os autores firmam que são componentes basilares da RUE:

As ações de saúde (Promoção, Prevenção e Vigilância à Saúde), Atenção Primária, Serviço de Atendimento Móvel de Urgência (SAMU), Sala de Estabilização, Força Nacional de Saúde do SUS, Unidades de Pronto Atendimento (UPA), Unidade Hospitalar e Atenção Domiciliar (CYRINO, 2017).

Neste sentido, dedicamos maior atenção ao componente balizar da Unidade Hospitalar, tendo em vista que para Sousa e Sousa (2018), a "unidade Hospitalar é vista na RUE como referência nas situações de maior gravidade dos usuários", com serviços especializados de Média e Alta Complexidade (MAC).

Diante das reformas político administrativas do Estado brasileiro, neste estudo, temos como objetivo principal mostrar os desafios da gestão com qualidade na prestação de serviços da área hospitalar, algo que faz parte do nível hierárquico terciário da RAS de Média e Alta Complexidade e que têm estabelecido padrões nas organizações de acreditação que avaliam a melhoria contínua, aplicada dentro das instituições de saúde. É possível constatar a difículdade de se entender a necessidade de intersecção entre as duas vertentes encontradas neste tipo de negócio (administrativo e o assistencial). São avaliadas as possibilidades e custos gerados no processo, além da preocupação em garantir a assistência adequada aos usuários dos serviços prestados

Com o intuito de agregar as informações necessárias para atingir a compreensão do tema abordado, realizamos revisão bibliográfica nos sites da Organização Nacional de Acreditação (ONA), da Associação Beneficente de Assistência Social Hospitalar (Pró-Saúde), do Google e em livros específicos da área. Corroborando com Deslanes $(2009$, p. 40$)$ que diz que, "o apoio de revisões bibliográficas sobre os estudos já feitos ajuda a mapear as perguntas já elaboradas naquela área do conhecimento", isso nos permite a identificação do "que mais tem sido enfatizado e o que tem sido pouco trabalhado".

A estrutura do artigo traz quatro tópicos: 1) Introdução, com um breve histórico do Sistema Único de Saúde a partir das reformas político administrativas em meados da década de 1980, além de apresentar o objetivo geral e metodologia; 2) Resultados e discussões, com a presença de nossos achados sobre ONA, desde a sua implantação, a disseminação da cultura de gestão com qualidade nas instituições hospitalares e análise dos resultados encontrados; 3) Considerações finais, que é balizada em nosso objeto geral. Trazemos nosso entendimento 
sobre os desafios da gestão com qualidade na prestação de serviços da área hospitalar e por fim, nossas referências.

Acreditamos que com essa estrutura nosso artigo contribuirá para a nossa compreensão sobre o objeto de estudo e que será de relevância na complementação dos estudos científicos existentes sobre a temática, tanto socialmente, como pessoalmente e academicamente.

\section{RESULTADOS E DISCUSSÕES}

Com a promulgação da Constituição Federal de 1988 que garantiu a Saúde como um direito de todos e dever do Estado, política social com "acesso universal e igualitário às ações e serviços para a promoção, proteção e recuperação da saúde", nasce o SUS. No Resumo sobre o Papel da Organização Nacional da Acreditação (RPONA) na construção do Sistema de Saúde brasileiro afirma-se que "a história da Organização Nacional da Acreditação está vinculada as iniciativas do SUS nos anos 1990" (ONA, s/a, p. 2).

No contexto brasileiro, antes da década de 1990, tais "discussões sobre acreditação e qualidade ocorriam de forma isolada, sendo que muitos não entendiam ainda o conceito de acreditação". A partir dessa década, com acordos firmados entre a Organização Mundial da Saúde (OMS) e a Organização Pan-Americana de Saúde (OPAS) há a elaboração "de um Manual de Padrões de Acreditação para América Latina e Caribe", tendo em sua concepção a "parceria entre o médico brasileiro Humberto Novais e o argentino José Maria Paganini, então dirigentes da OPAS." (ONA, p. 2).

Conforme registro no site oficial da Organização Nacional de Acreditação (ONA), sua constituição jurídica deu-se em junho de 1999. Em 2000, foi realizada a estruturação do Sistema Brasileiro de Acreditação e credenciamento das primeiras Instituições Acreditadoras. O lançamento do Manual Brasileiro de Acreditação, a realização de auditoria e concessão do selo ao primeiro hospital acreditado ocorreu em 2001. Em aproximadamente 20 anos de história, a ONA atingiu a marca de 800 certificações válidas.

A ONA “é responsável pelo desenvolvimento e gestão dos padrões brasileiros de qualidade e segurança em saúde" e "trabalha para que as instituições de saúde no Brasil adotem práticas de gestão e assistenciais que levem à melhoria do cuidado para o paciente", sendo responsável por $80 \%$ das instituições acreditadas no país. Tem reconhecimento internacional, pois é "membro da International Society for Quality in Health Care (ISQua)" que atua em conjunto com as "instituições da saúde que promovem a qualidade da saúde em países como Estados Unidos, Reino Unido, França e Canadá” (ONA, 2019, p. 1). 
BANDEIRA, J.A.A; BANDEIRA, M. A., Gestão Hospitalar: os desafios na implantação com qualidade. R. Científica UBM - Barra Mansa (RJ), ano XXVI, v. 23, n. 44, 1 . Sem. 2021 p. 103-114.

ISSN 1516-4071

A ONA atua com padrões de qualidade que podem ser adotados pelos seguintes serviços (ONA, 2019, p. 1):

- Hospitais;

- Ambulatórios;

- Laboratórios;

- Serviços de Pronto Atendimento;

- Home Care;

- Serviços Oncológicos;

- Serviços de Medicina Hiperbárica;

- Serviços de Hemoterapia;

- Serviços de Nefrologia e Terapia Renal Substitutiva;

- Serviços de Diagnóstico por Imagem, Radioterapia e Medicina Nuclear;

- Serviços Odontológicos;

- Serviços de Processamento de Roupas para a Saúde;

- Serviços de Dietoterapia;

- Serviços de Manipulação;

- Serviços de Esterilização e Reprocessamento de Materiais.

As instituições de saúde que se propõem ao desafio de Acreditação devem estar dispostas a investir no âmbito de reestruturação dos departamentos, recursos humanos e financeiros. Por meio do processo ocorrem mudanças na cultura organizacional e nos processos da organização. O desafio de gerir uma instituição de saúde baseada na qualidade dos serviços demanda total dedicação dos colaboradores, desde a alta gerência até o operacional. É importante que todos os envolvidos possuam a mesma visão, se empenhem na busca de um só objetivo: a Gestão com qualidade e excelência.

A gestão com qualidade traz para as instituições uma consequente mudança organizacional, pois exige a integração e participação de todos os seus integrantes. O processo de Acreditação traz uma cultura de melhoria contínua, em que se busca aprimorar a execução das atividades e trazer soluções aos problemas encontrados, através de treinamentos, workshop, auditorias internas e externas, análise dos indicadores de resultados.

A busca pela excelência dos serviços prestados traz como premissa a dedicação à qualidade das atividades, ressaltamos que "Um produto ou serviço de qualidade é aquele que atende perfeitamente, de forma confiável, de forma acessível, de forma segura e no tempo certo as necessidades do cliente." (CAMPOS, 1992, p. 2).

Algumas ferramentas básicas são utilizadas no processo de gestão com qualidade, como exemplo temos: 
- Diagrama de Pareto - Segundo Werkema (1995, p. 43) “o Diagrama de Pareto é um gráfico de barras verticais que dispõe a informação de forma a tornar evidente e visual a priorização de temas".

- Diagrama de causa e efeito - Segundo Maximiano (2009, p. 141) “o diagrama que tem a forma de uma espinha de peixe é um gráfico cuja finalidade é organizar o raciocínio e a discussão sobre as causas de um problema prioritário de qualidade".

- Estratificação - Segundo Werkema (1995, p. 42) “consiste no agrupamento da informação (dados) sob vários pontos de vista, de modo a focalizar a ação. Os fatores: equipamento, material, operador, tempo, entre outros, são categorias dos dados”.

- Folha de verificação - Segundo Paranhos Filho (2012, p. 116) a "Folha de Verificação consiste em uma planilha preparada para o levantamento de dados de um determinado problema".

Algumas entidades filantrópicas como as Organizações Sociais (OS), por exemplo a Associação Beneficente de Assistência Social e Hospitalar (Pró-saúde), trazem a cultura da qualidade como parte de sua identidade, tendo como visão a gestão qualificada para a excelência assistencial ao paciente. A referida instituição cultiva nas unidades hospitalares sob sua administração o desenvolvimento do seu capital humano, o controle de metas, sejam contratuais ou internas, através das comissões como: Serviço de Controle de Infecção Hospitalar (SCIH), Comissão Interna de Prevenção de Acidentes (CIPA), Núcleo de Qualidade e Segurança do Paciente (NQSP), óbitos, farmácia, dentre outras.

Para a Pró-saúde (2020, p. 1), “A inteligência é o principal ativo” sendo assim, “está presente em todas as etapas da gestão". Nesta linha "segue as diretrizes estabelecidas pela governança corporativa e pela política de integridade da organização", que "é aplicada ao cumprimento das cláusulas contratuais e ao plano de trabalho estabelecido pelo ente contratante".

A mensuração dos objetivos e metas nas instituições ocorrem por meio de indicadores de desempenho do processo, no âmbito hospitalar podemos citar os seguintes: Taxa de Infecção Global (TIG), Taxa de Mortalidade (TM), quantitativo de atendimentos nas diversas áreas, dentre outros.

É importante que os gestores cultivem a qualidade não apenas com intuito de alcançar certificações ou premiações que auxiliem na captação de novos recursos e investimentos, mas também para demonstrar a evolução dos seus processos em busca de excelência. Deve-se aplicar os conceitos de efetividade, eficácia e eficiência com intuito de proporcionar a qualidade 
BANDEIRA, J.A.A; BANDEIRA, M. A., Gestão Hospitalar: os desafios na implantação com qualidade. R. Científica UBM - Barra Mansa (RJ), ano XXVI, v. 23, n. 44, 1 . Sem. 2021 p. 103-114.

ISSN 1516-4071

nos serviços prestados ao seu cliente, que neste ramo são os pacientes, tornando-se referência em seu meio de atuação.

Para Quinto Neto e Gastal (1997, p. 24):

\begin{abstract}
A Acreditação Hospitalar não se constitui numa auditoria ou procedimento de habilitação institucional, porquanto estas são as funções de governo. Ela é uma maneira consistente de regular a qualidade dos serviços de saúde na medida em que os parâmetros de avaliação se modificam ao longo do tempo, além de que, globalmente, o instrumento contém itens que são 'puxados' para cima da média, a fim de servir de estímulo para a contínua melhoria dos serviços.
\end{abstract}

Em busca da melhoria contínua, é possível identificar e avaliar as falhas nos processos, com desafios que devem ser ultrapassados durante os caminhos percorridos para atingir o objetivo da excelência. Na avaliação das atividades realizadas, é possível identificar problemas e desenvolver suas resoluções através de ações de melhoria ou, em alguns casos, mudança completa da forma como são realizadas as atividades. O objetivo primordial é a constante evolução na metodologia implantada, trilhando um caminho em busca da segurança, qualidade e humanização.

É evidente que ,para o corpo clínico de uma instituição, manter uma gestão com qualidade é garantir o melhor tratamento aos seus pacientes, independente dos custos gerados. Para os administradores, a variável financeira é uma das mais importantes. A maioria dos hospitais tem seus recursos limitados, levando em consideração a necessidade do gerenciamento de custos. É, muitas vezes, necessário buscar o caminho mais viável no tratamento dos seus pacientes. Este fator é primordial nas discordâncias dos profissionais envolvidos.

A participação efetiva dos médicos junto ao gerenciamento de uma instituição se torna, assim, essencial, pois tem como um dos principais objetivos propiciar o entendimento entre as partes, corpo clínico e gestão, trazendo propostas plausíveis e de acordo com sua realidade. É primordial a parceria gerada entre as partes envolvidas para que possam ser ultrapassadas as barreiras que podem dificultar o alcance das metas estabelecidas. As discussões constantes apenas trazem insatisfação geral e não propiciam o alcance de seus objetivos.

Quando tratamos como oferta de serviço o auxílio no tratamento de problemas de saúde, as organizações devem estar dispostas a dedicar intensamente as suas forças de trabalho, tanto no âmbito operacional quanto administrativo, para prestar a assistência hospitalar de alta qualidade. 


\section{CONSIDERAÇÕES FINAIS}

Todo o esforço e dedicação dos envolvidos em um processo de gestão com qualidade irão garantir uma melhor imagem da empresa no mercado e, em consequência, a captação de novos clientes para seus serviços. É evidente que não se trata de um processo simples e de fácil desenvolvimento; há necessidade de dedicação, comprometimento e investimento.

A falha na interrelação entre a equipe assistencial e administrativa pode trazer consequências decisivas no processo de gestão com qualidade, motivo pelo qual é de suma importância a compreensão de todos os envolvidos quanto ao objetivo da instituição, quanto aos processos de gestão de seus serviços visando ao desenvolvimento e melhoria contínua, buscando sair de sua zona de conforto por meio dos processos de Acreditação, os quais permitem uma visão ampla das falhas e uma melhor efetividade em ações corretivas, que resultam na excelências dos serviços prestados.

A constante busca de gestão por qualidade tem se tornado um dos requisitos de mercado, em que, o cliente se baseia para aquisição de um determinado produto ou serviço. Em vista disso, as instituições que utilizam a gestão por qualidade tendem a possuir algumas vantagens competitivas.

À medida que evidenciamos a sedimentação das práticas de gestão com qualidade, constatamos que o cliente amplia seus critérios de avaliação quanto a determinada organização, utilizando os benefícios ofertados pela qualidade e excelência nos serviços como fator determinante nas suas escolhas.

É possível constatarmos, no Brasil, o sucesso no entendimento entre as partes envolvidas na área hospitalar, pois é crescente o reconhecimento de instituições que têm êxito nos processos de acreditação. 
BANDEIRA, J.A.A; BANDEIRA, M. A., Gestão Hospitalar: os desafios na implantação com qualidade. R. Científica UBM - Barra Mansa (RJ), ano XXVI, v. 23, n. 44, 1 . Sem. 2021 p. 103-114.

ISSN 1516-4071

\section{REFERÊNCIAS}

BORBA, G. S.; KLIEMANN NETO, F. J. Gestão Hospitalar: identificação das práticas de aprendizagem existentes em hospitais. Saúde Soc. São Paulo, São Paulo, v. 17, n. 1, p. 4460, 2008. Disponível em: https://www.scielosp.org/scielo.php?pid=S010412902008000100005\&script=sci_arttext. Acesso em: 29 fev. 2020.

BRASIL, F. G.; CAPELLA, A. C. N. Os estudos das políticas públicas no Brasil: passado, presente e caminhos futuros da pesquisa sobre análise de políticas. Revista Política Hoje, v. 25 , n. 1, p. 71-90, 2016.

BRASIL. [Constituição Federal (1988)]. Constituição da República Federativa do Brasil. Brasília, DF: Senado, 1988.

BRASIL. Decreto n. 7.508, de 28 de junho de 2011. Regula a Lei no 8.080/1990. Diário Oficial da União: Seção 1, Brasília, DF: Senado, p. 1, 29 jun. 201.

BRASIL. Lei n. 8.080, de 19 de setembro de 1990. Lei Orgânica da Saúde. Dispõe sobre as condições para promoção, proteção e recuperação da saúde, a organização e o funcionamento dos serviços correspondentes e dá outras providências. Diário Oficial da União: Seção 1, Brasília, DF, p. 18055, 20 set. 1990. PL 3110/1989

BRASIL. Lei n. 8.142, de 28 de dezembro de 1990. Lei Orgânica do Controle Social em Saúde. Dispõe sobre a participação da comunidade na gestão do Sistema Único de Saúde SUS e sobre as transferências intergovernamentais de recursos financeiros na área da sáude e dá outras providências. Diário Oficial da União: Seção 1, Brasília, DF, p. 25694, 31 dez. 1990. PL 5995/1990

BRASIL. Portaria n. 4.271, de 30 de dezembro de 2010. Diretrizes da Rede Atenção em Saúde. Diário Oficial da União: Seção 1, Brasília, DF, p. 88, 31 dez. 2010.

BURMESTER, H. Gestão da qualidade hospitalar. 1. ed. São Paulo: Editora Saraiva, s/a.

DESLANDES, S. F. (Org.). Pesquisa social: teoria, método e criatividade. 28. ed. Petrópolis, RJ: Vozes, 2009.

ONA. Resumo sobre o Papel da Organização Nacional da Acreditação (ONA) na construção do Sistema de Saúde brasileiro. ONA 20 anos. Disponível em: https://www.ona.org.br/resumo. Acessado em: 29 fev. 2020.

ONA. Sobre a Organização Nacional de Acreditação. 2019. Disponível em: https://www.ona.org.br/quem-somos/sobre-a-ona. Acesso em: 29 fev. 2020.

PEREIRA, G. S.; PEREIRA, S. S. A importância da qualidade do serviço na gestão hospitalar. Rev. Eletrôn. Atualiza Saúde, Salvador, v. 1, n. 1, jan./jun. 2015. Disponível em: http://atualizarevista.com.br/wp-content/uploads/2015/01/A-IMPORTANCIA-DAQUALIDADE-DO-SERVICO-NA-GESTAO-HOSPITALAR-REVISTA-ATUALIZASAUDE-N1-V1.pdf. Acesso em: 29 fev. 2020. 
BANDEIRA, J.A.A; BANDEIRA, M. A., Gestão Hospitalar: os desafios na implantação com qualidade. R. Científica UBM - Barra Mansa (RJ), ano XXVI, v. 23, n. 44, 1 . Sem. 2021 p. 103-114. ISSN 1516-4071

PRÓ-SAUDE. Inteligência em gestão. Disponível em:

https://www.prosaude.org.br/inteligencia-em-gestao. Acesso em: 29 fev. 2020.

SILVA, R. A.; SILVA, O. R. Qualidade, padronização e certificação. Editora Intersaberes, 2017.

SOUSA, F. D. T.; SOUSA, A. L. P. M. Uma revisão sobre as redes de atenção à saúde no Brasil. Revista Científica Multidisciplinar Núcleo do Conhecimento, v. 1, ano 3, ed. 11, p. 57-75, nov. 2018. ISSN: 2448-0959. 